\title{
Organizational Myths: How Organizational Change is Misunderstood and Why it is So Rarely Successful
}

\author{
Sydney Engelberg \\ Schwartz Program in Nonprofit Management, School of Social Work, Hebrew University, Mount Scopus, Jerusalem 91905, Israel \\ *Corresponding Author: Sydney.Engelberg@mail.huji.ac.il
}

\begin{abstract}
Organizational Myths is a conceptual paper that examines how, as a result of largely being unaware of and ignoring the "dark side" of organizational behavior, managers unintentionally fail in most of their improvement efforts. This failure rate is confirmed by numerous studies, e.g. Kotter (1996). An understanding of dark side issues provides for a deeper understanding of organizational resilience, and enables managers to deal more successfully with the complexity of organizational life, learn from success and build on failures. These insights apply equally well to for-profit and non-profit organizations of all kinds. The ideas developed in this article are based on over 30 years of international consulting experience, interviews with managers in diverse companies and organizations, and extension of basic findings in behavioral economics.
\end{abstract}

Keywords Change Management; Organizational Resilience; Human Resource Management; Organizational Innovation

\section{Introduction}

A good deal of current theory and practice in Human Resource Management (HRM) derives from what might be termed naïve human relations' assumptions.

Many of those advocating "excellence", "good to great", re-engineering and six sigma, to name but a few recent management trends, tend in their work, frequently, to hold powerful though unexpressed and unaware values that affect their "programs" as well as themselves. To give an example, the fervor with which the industrial world and management adopted "quality" and Total Quality Management (TQM) was based more on faith than evidence. This example illustrates the tendency to assume that HRM practices will naturally result in positive desired change. At a recent Global Human Resources Management Conference in November, 2011, we see presentations such as "Job Performance Evaluation as a Basis for Organization Function Improvement", or the consulting company of one of the keynote speakers offering a session on "Leveraging the
Recession for Competitive Advantage: Recessions are never a welcome occurrence, yet there are ways to take advantage of the situation. Whether that is rethinking how work gets done, focusing on your core capabilities, or streamlining key business processes you can use it as a time to strengthen your organization."

These claims are hardly consistent with the fact that for so long managers (and academics) have asked why so many change and management initiatives fail. In 1996, John Kotter published "Leading Change."

Considered by many to be the seminal work in the field of change management, Kotter's research revealed that only 30 percent of change programs succeed.

Since the book's release, literally thousands of books and journal articles have been published on the topic, and courses dedicated to managing change are now part of many major MBA programs. Yet in 2008, a McKinsey survey of 3,199 executives around the world found, as Kotter did, that only one transformation in three succeeds. Other studies over the past ten years reveal remarkably similar results. It seems that, despite prolific output, the field of change management hasn't led to more successful change programs.

The simple answer to this failure is that organizational change is complex and anyone knowledgeable about Complexity Theory is well aware that complexity is never linear. Almost all the change literature, including Kotter's "Leading Change" presents change as linear (just follow these steps). The deeper answer is that most managers (and academics) misunderstand the true nature of organizations and the complexity of organizational resilience.

In this paper I will examine some of the underlying assumptions in the HRM world and show that they are primarily derived from a certain set of biases in favor of "positive" emotions and attributes. As a result, other aspects of human interaction and organizational life that involve "negative" emotions and attributes have been neglected, given only lip service attention, or are in subtle ways denigrated. These "negative" aspects and their outcomes can be called the "dark side" of organizational life. The concept of a "dark side" to organizational life is similar to the concept of the shadow as used in the psychology of C.G. Jung. In Jungian psychology, the shadow characterizes those aspects 
of an individual personality that one has not accepted and integrated into one's consciousness and self-image. The shadow thus contains characteristics that often appear negative, alien, and threatening to the individual and they are frequently rejected. Nonetheless, in denying and disowning them one denies and disowns part of oneself. Since the struggle to repress the shadow can never be completely successful, one is also troubled by and at a loss as to how to cope with its intermittent appearance in daily life and behavior. The failure to openly address the dark aspects of HRM programs I believe has been, and is, the source of a severe limitation in the understanding and implementation of such programs and of organizational resilience. This is not to say that I am either pessimistic in my views or that I believe successful organizational change cannot be achieved. I am stating that we cannot be Pollyannaish and naïve in our approach to organizational change and that success stems from first being aware of the complexity of organizational change in order to successfully cope with the difficulties and challenges. This paper, therefore, is comprised of two parts; the first, presents four basic assumptions of organizational life that highlight the "myths" that too many managers, consultant and academics hold and that lead, consequently, to misguided change efforts. The second describes six concrete examples of how mistaken assumptions, or "myths", result in the exact opposite of the desired change outcomes managers and change agents would like to achieve.

\section{Design/methodology /approach}

The author has spent over 30years consulting and doing hands-on research with international companies, governments, and non-profits focused on two things:

a. Understanding the structure and governance or management systems required to operate effectively.

b. Defining and developing organization wide change management ability.

\section{Findings}

The author identifies four assumptions or principles of organizational reality, resilience and change management.

\section{Practical implications}

This paper presents a deeper understanding to guide managers through the complexities of organizational change and introduces a framework for the governance or management system of organizational change.

\section{The True Nature of Organizations}

Any student of current literature on management will be aware of the unfailingly positive nature of the processes and emotions that are presented. Whether we are talking about the statisticians and engineers (Deming, 1982, 1986;
Feigenbaum, 1983; Taguchi, 1995) or the human relations and organization development specialists (Senge, 1990, 1999; Kotter, 1996, 2002; Goleman, 1998, 2001), the values of these "utopians" include:

- logic and rationality

- trust of, and openness toward, others

- collaboration and participation

- affection and responsiveness

- group interest.

A perfect example is, “...As inspiring and instructive as stories like these are, they are just a start. At the MIX, we dream of organizations that can spontaneously renew themselves - where the drama of change is not accompanied by the wrenching drama of a turnaround, where innovation pulses through every activity, where every individual feels inspired by noble goals, and where collaborative cultures breed intense involvement. But of course, these aren't just dreams: they are do-or-die challenges for every organization that hopes to thrive in the future." (Hamel and LaBarre, 2010)

While these values certainly seem appropriate and important in the management and development of effective organizations, I believe that the management literature has largely neglected values at the other end of the spectrum of human interaction. For example:

- manipulation and power abuse

- caution and reserve

- autonomy and separateness

- competition and aggressiveness

- dislike and resistance

- self-interest

In order to genuinely understand the nature of organizational life and resilience and the complexity described above, I propose the following four basic assumptions:

Assumption One: Rational structuring of an organization is a myth. The reality of an organization is in existing pockets of information, pockets of influence, and pockets of sabotage.

Any observer of organizations is astounded to find that the members of an organization keep on developing and evolving new processes for managing information, power, and deviance. The process of managing this changing and shifting subculture activity is a primary characteristic of organizational life. In large organizations, the membership realignments in power and information groups are very rapid. Promotions, new course developments, new markets, competition with other systems, national policies, etc. require a continuous realignment and reassignment among strategic and key persons in an organization, and these subgroups form around personal style, personal choices, personal values, and personal preferences of those who assume temporary leadership of these subgroups. It comes, therefore, as no surprise that HRM efforts that largely ignore this organizational reality all too often result in superficial change at best.

Assumption Two: Publicly announced decision rules 
are not followed in practice during decision-making. Rule-making processes follow observable patterns. Observations indicate that, (a) most major decisions are irreversible and those who make these decisions will defend them stubbornly; (b) most major decisions are based on optimum incomplete information - as the amount of available information increases, the amount of decision-making discretion decreases; and (c) the influence structure in an organization is a function of individuals' abilities to grab (or fill) the influence vacuum regardless of organizational hierarchy.

The implications of these observed rules in decision-making are two-fold: (a) that individual members low in the organizational hierarchy learn risk-taking skills and discretion; and (b) that individual members need to develop a modicum of trust with other members in the organization to minimize conflict within decision-making under conditions of incomplete information. Once again, HRM efforts as they move away from technical areas where optimum complete information exists run into enormous difficulties trying to cope with the actual processes of decision-making in so-called "soft" areas of organizational life.

Assumption Three: The process of learning assumes freedom for regressive behavior and for experimentation with non-adult behavior in adult situations. The assumption that adults always behave as adults in the organizational situation is questionable. How often have you seen adults sulk, show apathy and engage in temper tantrums? The question, therefore, is not to demand adult behavior; rather, it is to create climates in which learning and interactions with each other are non-threatening. Adults sulk, show apathy, engage in temper tantrums, and show childlike spontaneity. An organization needs to create a non-threatening culture in which such childlike responses are not punished, but channeled into innovative directions. The implementation of HRM programs often results not in mature, inspirational and uplifting behaviors but in exactly the opposite. Behavior that is childish, vindictive, demoralizing and, in general, disappointing.

Assumption Four: The development of trust and openness requires the management of both problems of disagreement and agreement. Contrary to much organizational theory, actual organizational life demonstrates that achieving agreement and consensus does not necessarily result in the development of trust and openness. Often the exact opposite is the result. The reason is that there are two kinds of conflict, real and deceptive:

a) Real conflict, involves real, substantive differences. Differences that can be empirically addressed, resolved on the basis of data or experience, and that are issue rather than personality driven.

b) Deceptive conflict consists of the hostile, negative blaming behavior that occurs when agreement is mismanaged. This kind of conflict is a defensive measure after agreement has resulted not in the expected success but in failure. Rather than accepting shared responsibility for the negative outcome of this particular agreement in an atmosphere of trust and openness, the tendency is to become frustrated, angry, irritated and dissatisfied, blaming one another and the organization as a whole.

\section{Dark Side Issues}

Having described the dark side of organizational life in general, we can now consider specific dark side issues of HRM. For the purposes of this paper, we will consider six such examples:

\section{Forced Continuous Improvement: It's Effect on Self-Image and Motivation}

Think about the failure of almost all performance management systems. This is hardly surprising given that one of the cardinal principles of HRM thinking is continuous improvement. The never ending race - as it has been called or the race without a finishing line. The principle may make sense for organizations, but what are we to make of it at an individual level? Is it realistic to expect that individuals will never reach a ceiling to their achievement? I would argue that not everyone can continuously improve. In fact, most people reach what is, for them, an optimal level of functioning and are content to remain that way. Furthermore, those people who do strive for continuous improvement inevitably find that the increments of change become smaller and smaller. The result, for those who are content and for those finding improvement increasingly more difficult, is inevitable disillusionment. When we add to this an environment that demands or "forces" continuous improvement, individuals who are unable to "deliver" view themselves as failures. Their self-esteem is affected and their self-image becomes increasingly negative.

\section{Pseudo-empowerment}

One of the bedrocks of HRM literature is that change stems from autonomy and empowerment. These efforts range from improvement initiatives to self-managed teams. Underlying their ability to succeed is the organizational decision to empower lower level workers to take over in whole, or in part, problem-solving and decision-making processes. Unfortunately, there are many examples of management relinquishing power all too reluctantly or not at all. This we term 'pseudo-empowerment'. It is those situations where workers are led to believe that they have power, have decision-making authority, only to find that when it comes to the crunch they do not have any power.

A good example of this is when teams are told that they are not simply improvement teams but self-managed teams and then are severely restricted to dealing with technical issues only. The tough issues of team membership, salary, 
employee appraisals, promotion and career development are all outside the limits of their authority. The outcome is almost always anger, frustration, and disillusionment.

\section{Continuous Competition Heading to Inevitable Win/Lose Work Environments}

Anyone who has worked at Intel will understand this. Employees who are required by their organizations to demonstrate continuous improvements often find themselves drawn into an environment of continuous competition. I demonstrate achievement by outperforming others. They in turn are drawn into the vicious cycle of attempting to outdo me to demonstrate their achievement in the following round of assessments. At the macro level this inevitably creates a work environment where the dominant value and prevailing feelings are of being locked in a win/lose or zero sum setting. This is hardly the spirit of improvement but rather of improvement out of control. The kind of HRM program that may be thought of as good for management but certainly not for the employees and the organization in the long-run. For once, let me use an academic example, namely the well known finding that where students rate their instructors there is an erosion of academic standards. Lecturers, under pressure to get good ratings which can help lead to raises and tenure, find that the way to achieve these ratings is to "dumb down" their courses. Lecturers who insist on high standards consistently get lower ratings, damaging their chances for promotions. This problem is compounded by grade inflation resulting in too many students thinking they are above average academically. Thirty years ago, only $12 \%$ of incoming American college freshman were straight-A students. Today, $34 \%$ are straight-A students. What has happened? Has someone been doing wholesale genetic engineering on the sly? You decide.

\section{Empowerment without Preparation, O.J.T. or Support}

Involve employees in change say all the gurus. So why do so many change efforts fail? In an earlier example we analyzed pseudo-empowerment. An equally problematic issue is empowerment without the necessary training, tools and means. It is the equivalent of throwing someone who has never had swimming lessons into the deep-end of the pool and waiting to see if they sink or swim. Particularly in the case of more complex change such as self-managed teams, the need to prepare them, train them, provide on-going coaching and support until they achieve performance maturity, is enormous. The necessary training is not only in tools and techniques, as is so often the case. Genuine empowerment is accompanied by powerful group dynamic processes. It is these processes that all too often overwhelm the team and cause dysfunction. Knowledge of SPC, for example, is hardly of help in coping with difficult, often painful group dynamic processes.

\section{Commitment without Limits}

The decision to enter into quality programs carries with it a call for commitment. Commitment to specific values, commitment to the organization and its goals, commitment to work demands. The question arises, is there a limit to this commitment? If benchmarking shows that the "competition" is providing 24 hour customer support, is that a commitment that should be demanded of employees without the addition of manpower? Should commitment to the organization come at the price of commitment to one's family? There is no easy resolution to these dilemmas. What is clear is the potential for exploitation. In one case that I encountered, employees of a high tech company were "expected" to come in to work at the start of the Gulf War. Those who for family or other reasons did not come to work, even for a day, were considered "traitors". This, ironically, at a company that prides itself on its HR policies. No wonder people are waking up to life/work balance issues.

\section{Retrenchment and Improvement Programs}

The continuous improvement logic argues that a successful organization is a flatter, leaner organization. Flatter and leaner means less employees. Do improvement efforts mean fewer jobs? No, goes the argument because success results in greater market share, increased "sales", organizational growth. Employees don't lose their jobs, they simply need to be flexible and open to reallocation. You may not remain in job A, however you can be in job B where there is more demand. Does this argument hold true? The answer is, not necessarily. Firstly it ignores all those employees who cannot be sufficiently flexible, or simply like to specialize and develop expertise in one area. Secondly, it ignores macro-economics. When the economy is in recession organizational growth is impossible. Flatter and leaner really does mean fewer jobs. The problem for improvement programs during economic recessions is two-fold. The first, is whether to reveal to employees at the outset that initiating such programs will result in job loss, almost a certainty for creating motivational problems. The second is when organizations do not tell employees and they work it out for themselves. In my experience, those who work it out for themselves are usually the best and the brightest. They immediately take defensive measures which often means either sabotaging the program or leaving for another organization at their time and choosing, rather than when the organization would have wanted them to leave.

\section{The Contribution of Behavioral Economics}

The dark side of organizational life is exacerbated by the findings of behavioral economics. The new field of Behavioral Economics, the fastest growing area in 
economics' research, has taught us to what extent human decision-making is emotional and irrational. The Israeli cognitive psychologist Daniel Kahneman won the Nobel Prize for economics in 2002 for his work in this area. To give but two examples, of all the documented cognitive distortions, over optimism is one of the most likely to lead people who make strategic decisions astray, because decisions with an element of risk - all strategic ones - have two essential components. The first is a judgment about the likelihood of a given outcome, the second a value or utility placed on it.

When judging the likelihood of potentially positive outcomes, human beings have an overwhelming tendency to be overoptimistic or overconfident: they think that the future will be great, especially for them. Almost all of us believe ourselves to be in the top 20 percent of the population when it comes to driving, pleasing a partner, or managing a business. In the making of strategic decisions, optimism not only generates unrealistic forecasts but also leads managers to underestimate future challenges more subtly - for instance, by ignoring the risk of a clash between sub-cultures on a project requiring collaboration.

Our second example is what is known as "champion bias". This is the tendency to make a decision not on the merits of the evidence but on the basis of the proposer's reputation in the eyes of the decision-makers. All too often the employees who know best are ignored in favor of the more "trusted" senior executive.

In short, human nature has a tendency to heavily bias decision-making.

\section{Conclusion}

Because of the crucial significance I ascribe to understanding the complexity of organizational resilience and incorporating an understanding of the dark side of organizational life as part of that complexity, I wish to conclude this paper by recommending avoidance of all naïveté about recommendations and prescriptions for how to act when dealing with HRM efforts. Such caution would be necessary regardless of the particular values underlying and justifying particular actions. We all nod wisely when someone says that there is usually a discrepancy between what people say and what they do. Similarly, it is a cliché to say that the road to hell is paved with good intentions. But in labeling it as a cliché, we tend to be unaware that we are providing a description and not an explanation. That good intentions (such as those subsumed in the phrase "Total Quality Management" so often produce contrary effects needs explanation, not documentation. Clichés, like statistical correlations, describe relationships, not cause and effect dynamics.

Agreement on values is easier to reach than agreement about the appropriateness of value-derived actions. This alone should caution one against the tendency, tempting and understandable, to assume that because HRM programs incorporate a set of values which should inform action, it is a set of values that ensures certain desired outcomes. The failure to resist this tempting oversimplification leads only to undesired outcomes and disillusionment. For this reason, recommendations such as, awareness of dark side issues and decision bias will provide the necessary insight, or have a designated "devil's advocate", or ensure frequent, rapid and unambiguous feedback are all good and well but hardly the panacea required to solve all difficulties.

Human Resource Management is a high-sounding phrase, an inspirational slogan. To be against it is to appear to be for sin and against virtue. The eagerness with which we accept it as an unalloyed "good" testifies more to the strength of our need for solutions to the problems plaguing our economies, organizational life and management achievements than to our understanding of the realities and complexity of achieving successful, stable, long-term organizational resilience, change and success. There is no formula for how to implement and maintain successful HRM. Indeed, the point of this paper is that before we indulge our tendency to develop formulas and techniques (to become absorbed with technical-engineering issues) or to turn to commercially packaged HRM "solutions" in our endeavors to effect successful change, we need to understand better how the nature of people and of everyday organizational life produce the situations we wish to change and create undesired outcomes despite our best intentions. We can learn a lot not only from success but from failure if we are able to see how we and the nature of our organizational life contribute to it. With increased awareness and understanding comes improved coping abilities and improved coping abilities allow us to facilitate change far more effectively.

\section{REFERENCES}

Deming, W. Edwards. Out of the Crisis. Massachusetts Institute of Technology, Center for Advanced Engineering Study, Cambridge, Mass., 1986.

Deming, W. Edwards. Quality Productivity and Competitive Position, Massachusetts Institute of Technology Center for Advanced Engineering Study, Cambridge, Mass., 1982.

Feigenbaum, Armand V. Total Quality Control. McGraw Hill Book Co., New York, 1983.

Goleman, Daniel. Working with Emotional Intelligence. Bantam Books, 1998.

Goleman, Daniel. The Emotionally Intelligent Workplace. Jossey-Bass, 2001.

Hamel, Gary and Polly LaBarre. Meet the M-Prize winners - three case studies in management innovation honored by Gary Hamel's Management Innovation eXchange. McKinsey Quarterly, November 2010. 
Kahneman, Daniel. "A perspective on judgment and choice: Mapping bounded rationality". American Psychologist 58 (9): 697-720, 2003.

Kotter, John P. Leading Change. Harvard Business Press, 1996.

Kotter, John P. and Dan S. Cohen. The Heart of Change. Harvard Business Press, 2002.

Senge, Peter. The Fifth Discipline: The Art and Practice of the
Learning Organization. Doubleday, 1990

Senge, Peter, et al. The Dance of Change: The Challenges to Sustaining Momentum in Learning Organizations. Doubleday, 1999.

Taguchi, Genichi "Quality engineering (Taguchi methods) for the development of electronic circuit technology". IEEE Transactions on Reliability (IEEE Reliability Society) 44 (2): 225-229, 1995. 